Edicion Extraordinaria.

morias del VIII Congreso y III Encuentro Nacional de Investigación en Enseñanza de la Biología y La Educación Ambiental. ISSN 2027 - 1034 P.p. 1802-1811

\title{
LA EDUCACIÓN AMBIENTAL EN LOS ESPACIOS NO CONVENCIONALES DE EDUCACIÓN QUE GERENCIAN LAS ENTIDADES QUE CONFORMAN EL SECTOR AMBIENTAL DE BOGOTÁ Y SU DIÁLOGO CON LA EDUCACIÓN FORMAL
}

\author{
THE ENVIRONMENTAL EDUCATION AT PLACES OF NOT CONVENTIONAL \\ EDUCATION THAT ARE MANAGED BY THE ENTITIES WHICH BUILD UP THE \\ ENVIRONMENTAL SECTOR IN BOGOTA AND ITS DIALOGUE WITH THE FORMAL \\ EDUCATION \\ Autor: Tania Elena Rodríguez
}

\section{RESUMEN}

Ante la necesidad de indagar sobre la forma como se asume e implementa la Educación Ambiental en los espacios no convencionales de educación que gerencian las entidades que conforman el sector ambiental de Bogotá, se hace necesario, a manera de referente conceptual, abordar las discusiones que se adelantan en el ámbito de la educación formal, sobre la "educación para el desarrollo sostenible" (como proyecto político internacional nacida en la Cumbre de la Tierra, Río de Janeiro Brasil, 1992), que ha generado desarrollos educativos que tradicionalmente habían sido del campo de la Educación Ambiental; como también la discusión internacional adelantada sobre las relaciones entre la Educación Ambiental y la Educación en Ciencias con el fin de analizar las maneras en que estas relaciones estratégicas permitan superar reduccionismos y desconfianzas entre estos tipos de educación. Así mismo, este trabajo intentará establecer la posible emergencia de una Didáctica Ambiental para espacios de educación no convencional, como un facilitador de diseño de contenidos desde actuales posturas teóricas sobre lo ambiental.

\section{SUMMARY}

Being in need of investigating about the way of how environmental education is being introduced in the non conventional education spaces that are managed by the Bogotá environmental entities, is necessary, by way of conceptual approach, the discussion made in the field of non formal education, about the "education for sustainable development" (as international political project born in the earth summit, Rio de Janeiro Brazil, 1992), this has generated educational developments that had traditionally been the field of environmental education; as well as the international discussion about the relationship between environmental education and science education to analize the ways in which strategic relation help overcome reductionism and distrusts between these types of education. Also, this study intends to establish the possible emergence of environmental didactic for non conventional education spaces as facilitator of content design from current theoretical views about environmental. 
Edicion Extraordinaria.

\section{Memorias del VIII Congreso y III Encuentro Nacional de Investigación en Enseñanza de la Biología y} La Educación Ambiental. ISSN 2027 - 1034 P.p. 1802-1811

Educación Ambiental, educación para el desarrollo sostenible, educación en ciencias, didáctica ambiental, Jardines Botánicos.

\section{KEYWORDS}

Environmental education, education for sustainable development, science education, environmental didactic, botanical gardens.

\section{INTRODUCCIÓN}

El interés en abordar el tema de la Educación Ambiental (EA) en los espacios no convencionales de educación que gerencian las entidades que conforman el sector ambiental de Bogotá, nace en el marco del contexto formativo doctoral en educación (en la línea de investigación de articulación entre EA y Educación en Ciencias (EC), y a partir de haber hecho un largo recorrido en la EA. Preguntas como las siguientes han emergido en 16 años como estudiante y 19 en el ejercicio profesional docente y administrativo, las cuales guían éste interés investigativo, pero no todas serán abordadas en el desarrollo de la tesis doctoral:

\section{actual empeora?}

¿Por qué, después de muchos esfuerzos y tiempo invertido, la situación ambiental ¿Qué puede hacer la escuela frente a la crisis ambiental latente como espacio donde confluyen múltiples interpretaciones de la realidad por parte de los diferentes actores que la constituyen?

¿Cómo redimir una EA criticada y subvalorada por una EC idealizada? y en consecuencia ¿Cómo generar análisis crítico ambiental para superar estas creencias y su fácil reproducción?

¿Qué relaciones deseables en el marco de la EA, se pueden establecer entre la educación formal y la informal especialmente con los espacios de educación no convencional gerenciados por las entidades que conforman el sector ambiental de Bogotá?

¿Cuál deberá ser el enfoque educativo que permita promover la reflexión de lo ambiental y dinamice un cambio en las visiones heredadas y dominantes existentes en la actualidad?

¿Cómo lograr que los próximos gestores del cambio ambiental se empoderen, resistan y reconfiguren un sistema que tradicionalmente ha sido jerarquizado, dominante y excluyente? ¿Cómo proponer "políticas arriesgadas" que transformen la manera de ver, sentir, hacer, y evaluar la realidad ambiental?

Estas preguntas, que hacen parte de la reflexión que se está dando a nivel internacional sobre la EA, le abren paso a preguntas del contexto más local que surgen específicamente desde mi experiencia laboral en el Jardín Botánico José Celestino Mutis (JBJCM), entidad que junto con la Secretaría Distrital de Ambiente (SDA) conforman el sector ambiental de Bogotá, responsable de liderar la formulación de políticas, la gestión y la EA en el Distrito Capital.

Dicho proyecto de investigación doctoral además intentará establecer relaciones entre las formas de asumir e implementar la EA en el ámbito de lo formal e informal y la posible 
Edicion Extraordinaria.

Memorias del VIII Congreso y III Encuentro Nacional de Investigación en Enseñanza de la Biología y La Educación Ambiental. ISSN 2027 - 1034 P.p. 1802-1811

emergencia de una Didáctica Ambiental para dichos espacios, como un facilitador conceptual en la reflexión crítica acerca de las vigentes bases conceptuales de la EA y las actuales posturas teóricas sobre lo ambiental.

De acuerdo con lo anterior y por encontrarse el proyecto doctoral es su primera fase de formulación, la comunicación que se presenta a continuación centra su atención en la necesidad de indagar sobre la forma como se asume e implementa la EA en los espacios no convencionales de educación, que gerencian las entidades que conforman el sector ambiental de Bogotá, analizada dicha necesidad desde la intencionalidad y conveniencia de la investigación y presentada en tres ejes: el situacional, el problémico y el relacional.

Se espera que en posteriores encuentros se pueda presentar la metodología, los resultados y las conclusiones de éste proyecto comprometido con la educación y el ambiente de Bogotá.

El eje de Intencionalidad y conveniencia de la investigación: Los problemas identificados.

La pregunta que subyace a la presente investigación es ¿cómo se está entendiendo e implementando la EA en el ámbito de la relación entre educación formal e informal en el D.C?, para intentar aportar a la respuesta de esta pregunta macro, es necesario operacionalizarla desde el eje situacional de la misma (los espacios no convencionales de educación gerenciados por el sector ambiental de Bogotá), y mediante un ejercicio de reflexión problémica, se da como resultado: 1) el planteamiento de tres problemas centrales (pc) que derivan en tres preguntas orientadoras (po) las cuales dan forma a los ejes de la investigación, y 2) unos problemas auxiliares (pa), que al ser resueltos, le darán sentido a los problemas centrales:

- Pc1: No se ha profundizado empíricamente sobre las maneras en que las entidades del sector ambiental de Bogotá entienden la EA.

- Po1: ¿Cómo se está entendiendo e implementando la EA desde los espacios no convencionales de educación que son gerenciados por las entidades que conforman el sector ambiental de Bogotá? (educación informal)

- Pc2: No existe una propuesta de EA integradora y coherente entre los ámbitos de educación formal e informal.

- Po2: ¿Cómo establecer relaciones entre las formas de asumir e implementar la EA en estos espacios y la educación formal?

- Pc3: No existe un facilitador conceptual entre la EA, la EDS y la EC pensado desde la educación formal e informal

- Po3: ¿Cómo plantear una emergente "Didáctica Ambiental" para los espacios no convencionales de educación que son gerenciados por las entidades que conforman el sector ambiental de Bogotá, propuesto como un facilitador conceptual y fruto de la reflexión crítica acerca de las actuales bases conceptuales de la EA y las posturas teóricas actuales sobre lo ambiental?

Los problemas auxiliares serían: 
Edicion Extraordinaria.

Memorias del VIII Congreso y III Encuentro Nacional de Investigación en Enseñanza de la Biología y La Educación Ambiental. ISSN 2027 - 1034 P.p. 1802-1811

- pa1: ¿Son las concepciones de EA existentes en los espacios no convencionales de educación gerenciados por las entidades que conforman el sector ambiental de Bogotá, potencialmente adecuadas para los procesos de integración demandados?

- pa2: Cómo afecta teóricamente el hecho que en las políticas internacionales la EA esté subsumida o desplazada por la EDS

- pa3: Qué efectos podrían tener visiones y prácticas de desconfianza y minimización de la EA en relación con la EC.

\section{El eje situacional de la investigación. Po1}

El común denominador de muchas investigaciones encontradas en bases de datos especializadas ${ }^{1}$, bajo el tesauro -Environmental outdoor education-, es que se evidencia una clara propuesta de uso de estos espacios no convencionales de educación por parte de la educación formal centrada en el aprendizaje de los contenidos del currículo de ciencias, lo que abre un horizonte prometedor para el presente proyecto de investigación.

A nivel Nacional, sucede algo parecido. En la investigación de Zambrano et al (2013), se presenta la línea de investigación "Contextos culturales - Educación ambiental Educación en Ciencias en Ambientes no Convencionales" (Zambrano et al., 2007, pág. 98). Allí en la Universidad de Antioquia la profesora Fanny Angulo ha presentado avances sobre "la importancia que juegan los ambientes no convencionales, en la enseñanza y aprendizaje de los contenidos del currículo de ciencias" (p.99).

Lo anterior permite concebir la reflexión desde un contexto más local y específico para el presente interés investigativo, por ello, se hace a continuación una corta descripción sobre el JBJCM y la SDA, entidades que cuentan con dinámicas propias, con proyectos de inversión que reciben recursos del Distrito y con concepciones construidas desde dos ejes: la experiencia y el conocimiento de la realidad ambiental de Bogotá y las reflexiones gnoseológicas $^{2}$ que se han dado hasta el momento.

EL JBJCM como Entidad Distrital se define como centro de investigación y desarrollo científico en donde hace parte de su misión la EA (JBJCM,2014). Para el periodo 2012-2016, la EA se formula como una estrategia en el marco de un proyecto de inversión del plan de desarrollo Bogotá Humana, el cual está orientado hacia la armonización de las relaciones ecosistema - Cultura pero con la especificidad de reducir la vulnerabilidad frente al cambio climático (JBJCM, 2012). Dicha estrategia de EA plantea seis líneas de acción así:

\begin{tabular}{c|c|}
\hline Línea de acción & Descripción \\
$\begin{array}{c}\text { Pensamiento Ambiental } \\
\text { Intercultural }\end{array}$ & $\begin{array}{l}\text { Se realizan encuentros de diálogo y entendimiento intercultural entre distintos actores de la región } \\
\text { capital, que contribuyan a la configuración de un tejido de visiones, experiencias y estrategias para la }\end{array}$ \\
\hline
\end{tabular}

\footnotetext{
${ }^{1}$ se adelantó la revisión de 683 abstrac de artículos escritos en este campo.

${ }^{2}$ Gnoseología como enfoque propuesto por Martínez A; Vasco M.E; y Vasco U.C. (2008) entendida más como la reflexión filosófica sobre aquellos saberes difusos propios de las prácticas o discursos que aún no han transitado hacia una epistemologización (de nociones a conceptos / de opiniones a teorías).
} 


\author{
Edicion Extraordinaria.
}

\title{
Memorias del VIII Congreso y III Encuentro Nacional de Investigación en Enseñanza de la Biología y La Educación Ambiental. ISSN 2027 - 1034 P.p. 1802-1811
}

\begin{tabular}{|c|l|}
\hline Interpretación Ambiental & protección, cuidado y conservación de la biodiversidad. \\
\hline $\begin{array}{c}\text { Busca poyar los procesos de enseñanza-aprendizaje realizados desde el sistema educativo formal: } \\
\text { preescolar, básica y secundaria y los realizados desde el sistema de educación superior, proponiendo } \\
\text { ampliar los contenidos para incrementar el número de visitantes universitarios y el área de disciplinas } \\
\text { que visitan el Jardín. Así mismo busca el posicionamiento del JBJCM, como espacio ideal para el } \\
\text { turismo científico, cultural y sostenible. }\end{array}$ \\
\hline $\begin{array}{c}\text { Transversalización de la } \\
\text { dimensión ambiental en la } \\
\text { educación formal }\end{array}$ & $\begin{array}{l}\text { Busca orientar procesos de transversalización de la dimensión ambiental en colegios, proponiendo } \\
\text { elementos conceptuales y metodológicos en la apropiación del territorio y la gobernanza del agua y la } \\
\text { orientación de procesos de EA en diferentes niveles de formación. }\end{array}$ \\
\hline $\begin{array}{c}\text { Semilleros para el } \\
\text { reconocimiento territorial }\end{array}$ & $\begin{array}{l}\text { Conformación de grupos de investigación dentro de comunidades organizadas o Instituciones } \\
\text { Educativas. }\end{array}$ \\
\hline $\begin{array}{c}\text { Articulación de procesos } \\
\text { comunitarios de EA }\end{array}$ & $\begin{array}{l}\text { Pretende articular iniciativas de las comunidades organizadas en torno a la EA, alrededor de las cuencas } \\
\text { del D.C. y los páramos para disminuir las vulnerabilidades frente al cambio climático. }\end{array}$ \\
\hline Análisis de políticas de EA & $\begin{array}{l}\text { Se espera determinar las fortalezas y debilidades frente a la implementación de las políticas nacional y } \\
\text { distrital de EA, para proponer estrategias que permitan fortalecer el sistema distrital ambiental. }\end{array}$ \\
\hline
\end{tabular}

Tabla 1. Información tomada del proyecto de inversión 865 del JBJCM. "Armonización de las relaciones ecosistema - Cultura pero con la especificidad de reducir la vulnerabilidad frente al cambio climático"

En el periodo 2012-2016 para la SDA, la EA se entiende como "un instrumento de gestión para la apropiación social de los territorios ambientales del Distrito Capital en el marco de la gobernanza del agua y la recuperación de la estructura ecológica principal" (SDA,2014,p.11), descrita en el proyecto de inversión denominado participación ciudadana y educación ambiental como instrumentos de gestión para la apropiación social de los territorios ambientales del D.C. y consolidada en el diseño dos grandes líneas de acción, así:

\begin{tabular}{|l|l|}
\hline \multicolumn{1}{|c|}{ Línea de acción } & \multicolumn{1}{c|}{ Descripción } \\
\hline $\begin{array}{l}\text { Implementación de la Política Pública Distrital } \\
\text { de Educación Ambiental }\end{array}$ & $\begin{array}{l}\text { Esta línea se ejecuta a través de las siguientes actividades: } \\
\text { * Vinculación de la ciudadanía en las estrategias de EA priorizadas por cada } \\
\text { localidad en el marco de la PPDEA. } \\
{ }^{*} \text { Ejecución de la estrategia de aulas ambientales en el Distrito Capital, Parque } \\
\text { Ecológico Distrital de Montaña Entre nubes, Parque Mirador de los Nevados, } \\
\text { Parque Ambiental Soratama y Parque ecológico Distrital Humedal Santa María del } \\
\text { Lago. }\end{array}$ \\
$\begin{array}{l}{ }^{*} \text { Fortalecimiento de la Comisión Intersectorial de EA, CIDEA y ejecución de la } \\
\text { agenda de trabajo. }\end{array}$ \\
$\begin{array}{l}{ }^{*} \text { Ejecución de los programas y líneas de acción de la PPDEA. } \\
\text { Apoyo a la gestión ambiental Distrital para el } \\
\text { fortalecimiento de la participación comunitaria } \\
\text { y la vinculación de organizaciones ambientales } \\
\text { del Distrito en los procesos de conservación } \\
\text { de los territorios ambientales que componen la } \\
\text { estructura ecológica principal. }\end{array}$ & $\begin{array}{l}\text { Apoyo continuo a las diferentes organizaciones ambientales del Distrito, en el } \\
\text { fortalecimiento y vinculación de la participación ciudadana para abordar temas } \\
\text { como el ordenamiento del territorio alrededor del agua o la gestión integral de } \\
\text { riesgos y desastres y la adaptabilidad al cambio climático, mejorando de esta } \\
\text { manera la capacidad de gestión desde las localidades hacia la conservación de los } \\
\text { territorios ambientales que comprende la Estructura Ecológica Principal. }\end{array}$ \\
\hline
\end{tabular}

Tabla 2. Información tomada del proyecto de inversión 131 de la SDA. "Participación ciudadana y educación ambiental como instrumentos de gestión para la apropiación social de los territorios ambientales del Distrito Capital"

De acuerdo con lo anterior, la presente investigación centra su interés en dos líneas de acción, una es la línea de acción interpretación ambiental del proyecto 865 del JBJCM y la segunda, la línea de acción Implementación de la Política Pública Distrital de Educación Ambiental del proyecto 131 de la SDA, debido a que comparten un común denominador:

1) Se desarrollan en los espacios de educación no convencional propios del JBJCM y los de la SDA como son sus cuatro aulas ambientales (Parques Mirador de los Nevados y 
Edicion Extraordinaria.

\section{Memorias del VIII Congreso y III Encuentro Nacional de Investigación en Enseñanza de la Biología y La Educación Ambiental. ISSN 2027 - 1034 P.p. 1802-1811}

Soratama, el Parque Ecológico Distrital de Humedal Santa María del Lago y el Parque Ecológico Distrital de Montaña Entrenubes)

2) La población que los visita anualmente es numerosa de diferentes edades, genero, etnia, estrato y nivel educativo, destacándose los estudiantes y docentes de colegios oficiales o privados de la ciudad, lo que posibilita el encuentro entre la educación formal y la informal.

3) Estos espacios no convencionales de educación permanecerán en la ciudad y seguirán siendo escenarios de la EA, independientemente del plan de desarrollo vigente y en consecuencia del proyecto de inversión formulado.

Los aspectos que se mencionan a continuación, hacen parte de una lectura que se realiza basada en la experiencia laboral del investigador sobre la implementación de la línea de acción interpretación ambiental del JBJCM, e invitan a la reflexión crítica sobre cómo se entiende y se aborda la EA desde estos espacios gerenciados por las entidades que conforman el sector ambiental de Bogotá:

a. Las actividades propuestas se desarrollan a través de programas como: Club de ciencias, recorridos guiados y ecotalleres, los cuales se estructuran a partir de los estándares curriculares en ciencias (para grupos escolarizados) en donde el eje es la comprensión de los mismos utilizando la colección viva del JBJCM y "metodologías con materiales didácticos de apoyo, como parte del aprendizaje de las ciencias y el proceso de sensibilización frente a la problemática ambiental actual" (JBJCM, 2014, p.114).

b. Los fines de semana se realizan recorridos guiados para dar a conocer los diferentes ambientes del JBJCM.

c. El tiempo designado a cada una de las actividades es de 2 horas, a excepción del club de ciencias que se reúne los sábados, medio día durante un semestre.

d. Existe un primer encuentro con el líder del grupo que reserva su visita al JBJCM quien escoge según su interés particular, alguno de los programas ofertados y se establecen recomendaciones sobre la visita.

e. Los grupos se conforman de 20 a 30 personas y si es de carácter escolar, deben ir acompañados por un docente.

f. Las situaciones que se dan durante el desarrollo de la actividad programada, es que los docentes acompañantes generalmente no intervienen o delegan por completo la responsabilidad al profesional del JBJCM, son pocos los docentes que con sus aportes enriquecen la actividad. Algunos grupos pueden ingresar solos, pero estos son de carácter universitario.

g. Existe una encuesta de satisfacción de la visita.

h. La posibilidad de encontrarse más de una vez con el mismo grupo en el año es casi remota.

i. Las metas formuladas en el plan de acción de la entidad, los cuales responden a los lineamientos del plan de desarrollo vigente, así como los indicadores planteados para el seguimiento de las mismas, se establecen bajo el concepto de eficacia y eficiencia de los procesos. Las metas cuantitativas de la línea se establecen en el sentido de 
Edicion Extraordinaria.

\section{Memorias del VIII Congreso y III Encuentro Nacional de Investigación en Enseñanza de la Biología y La Educación Ambiental. ISSN 2027 - 1034 P.p. 1802-1811}

facilitar 0 atender $X$ número de personas en actividades de interpretación ambiental. (JBJCM, 2014, p.107-111).

En lo que respecta a la línea de acción Implementación de la Política Pública Distrital de Educación Ambiental de la SDA, una de sus actividades se denomina "Ejecución de la estrategia de aulas ambientales en el Distrito Capital" (SDA, 2014. P.12), y aunque no se cuenta con una experiencia cercana a la propuesta, se puede intuir que a excepción del punto (a) los demás ítem pueden describir una situación parecida. El hacer partícipe a la SDA en la reflexión sobre las realidades que se viven en estos espacios, puede propiciar la construcción conjunta de lineamientos de política, lo que sería un importante efecto colateral en esta investigación.

\section{El eje problémico de la investigación: Po2}

Pensar en la EA desde la política internacional, más específicamente desde la Conferencia de las Naciones Unidas sobre Medio Ambiente y Desarrollo (Cumbre de la Tierra, en Río de Janeiro en junio de 1992), y su agenda 21, es pensar en una educación que ya no se referencia como ambiental sino como "Educación para el desarrollo sostenible (EDS)"3.

A partir de allí la EA pasa a ser una educación que responde a diferentes intereses desdibujando su naturaleza misma, aunque la reflexión que se hace en algunos lugares de Latinoamérica comienza a cuestionar lo que subyace a una EDS, en Colombia, la Política Nacional de EA en su objetivo general orienta su accionar hacia "el manejo sostenible del ambiente" (MAVDT \& MEN, 2002, p.27), aunque por su lado, la Política Distrital de EA se orienta más hacia una consolidación de una "ética ambiental en el marco del desarrollo humano integral' (SDA \& SDE,2007,p.39).

Así mismo, en el ámbito de la educación formal, se le ha adjudicado a los docentes que lideran la EC (biología, física y química) la responsabilidad de asumir la EA, tal vez sin considerar que la EC y la EA no tienen el mismo estatuto epistemológico, la misma importancia cultural y educativa, o el mismo tiempo de haber surgido, lo que puede generar tensiones y presentar dificultades al intentar relacionarlos. De acuerdo con lo anterior, Michael Littledyke (1997), presenta los resultados de una etapa preliminar de su investigación en donde concluye que intentar incluir la EA en el currículo presenta como obstáculos el contenido extenso que se aborda desde la EC y las presiones de cumplir el plan de estudios lo que conlleva a tener una EA subvalorada y relegada fuera de un plan de estudios saturado de otras prioridades.

Resulta que en este enfrentamiento, la EA es la que pierde la partida, así se puede evidenciar en las investigaciones adelantadas por Lucié Sauvé (2014) ${ }^{4}$, quien al indagar las concepciones de los profesores que enseñan ciencias, encontró los que piensan que la EA es bienvenida en la EC ya que el medio ambiente es buen pretexto, un buen disparador de

\footnotetext{
${ }^{3}$ http://www.un.org/spanish/esa/sustdev/agenda21/agenda21spchapter36.htm “Capítulo 36. Fomento de la Educación, la capacitación y la toma de conciencia. Programa: Reorientación de la educación hacia el desarrollo sostenible"

${ }^{4}$ En conferencia realizada el 6 de Octubre del 2014 en el marco del Doctorado Interinstitucional de Educación en Bogotá. Colombia.
} 
Edicion Extraordinaria.

\section{Memorias del VIII Congreso y III Encuentro Nacional de Investigación en Enseñanza de la Biología y La Educación Ambiental. ISSN 2027 - 1034 P.p. 1802-1811}

interés en el estudio científico otorgándole de hecho a la EA, una finalidad motivadora para el aprendizaje de las ciencias. El panorama no mejora para la EA cuando se encuentran profesores que piensan que ésta es una amenaza a la EC ya que se corre el riesgo de que pierda su contenido disciplinario en tanto que educar en valores no es ciencia.

Investigadores como Margarita Bown, Annette Gough, Funtowicz\&Ravetz, Lucié Sauvé, William Mora entre otros, ven la necesidad de fortalecer esta relación proponiendo nuevos enfoques educativos como una ecociencia ó una ciencia posnormal ó una educación Ecocientífica, o una educación científica ambientalizada, con la idea de proponer unas "ciencias escolares más pertinentes y acordes a las problemáticas socio ambientales que más afectan al mundo en la actualidad" (Mora, 2013, p.136).

En concordancia con lo expuesto, los aportes y reflexiones adelantadas en el ámbito de la educación formal, sobre la "educación para el desarrollo sostenible" y la discusión adelantada sobre las relaciones entre la EA y la EC, se entienden en este proyecto de investigación como el referente necesario para reflexionar críticamente sobre la EA, puesto que, lo que sucede en el ámbito de la educación formal se puede reflejar en la forma como el sector ambiente asume la EA en sus espacios, al ser estudiantes y docentes de los colegios de la ciudad, sus visitantes por excelencia, y por supuesto en la posibilidad de encontrar las relaciones entre las formas de entender y asumir la EA, pregunta central del eje problémico de la investigación.

\section{El eje articulador: Po3}

La reflexión crítica acerca de las vigentes bases conceptuales de la EA y las posturas teóricas actuales sobre lo ambiental se entiende en esta investigación como una categoría de estudio que le permite al investigador tomar una postura epistemológica frente a lo ambiental creando un compromiso teórico y no como un objeto de investigación en sí mismo, es decir, se entiende como dispositivo potenciador del análisis conceptual y teórico, mas no empírico.

Es así como dicha reflexión se convierte en la aguja que teje los hilos proporcionados en el diálogo con algunos autores que reflexionan sobre lo ambiental (más propios de la postmodernidad), en el ideal de tejer una emergente didáctica ambiental pensada desde y para los espacios no convencionales de educación que son gerenciados por las entidades que conforman el sector ambiental de Bogotá.

Autores como Leff (2007) y la aproximación entre diferentes en donde debe aludirse a la otredad y la alteridad surgiendo el diálogo de saberes y su concepto de cultura, Morin (1996) y el pensamiento complejo, Elizalde (2006) y el concepto de sustentabilidad; Sauvé (2010) y el escenario político como postura clara de lo ambiental y lo educativo, los sistemas sociales autorreferentes de Luhmann (1996); Freire y su postura sobre el poder y la alfabetización crítica, Boaventura de Sousa (2013) y su reinvención del poder desde la epistemología del sur, son algunos autores, solo algunos entre muchos otros, que serán invitados a la reflexión con el fin de asumir el reto de pensar y pensarnos lo ambiental de 
Edicion Extraordinaria.

\section{Memorias del VIII Congreso y III Encuentro Nacional de Investigación en Enseñanza de la Biología y La Educación Ambiental. ISSN 2027 - 1034 P.p. 1802-1811}

forma diferente y porque no, desde una perspectiva discursiva que no es solo lenguaje, que es lenguaje y práctica.

\section{BIBLIOGRAFIA}

La bibliografía que se presenta a continuación es la referenciada en la comunicación anterior, la cual es rigurosa, coherente y pertinente para los fines de la misma, sin embargo es importante mencionar que para construir el documento se consultaron autores adicionales que hacen parte del estado del arte de la investigación.

Boaventura de Sousa, S. (2013). Descolonizar el saber, reinventar el poder. Santiago: LOM Ediciones.

Elizalde, A. (2006) Desarrollo Humano y ética para la sustentabilidad. Medellín: Universidad de Antioquia.

Freire, P \& Macedo, D.(1989). Alfabetización. Lectura de la palabra y lectura de la realidad. Barcelona: Paidós.

JBJCM (2014). Informe de Gestión. Recuperado de http://www.jbb.gov.co/jardin/institucionalinicio/informes-e-indicadores- de-gestion

JBJCM. (2012). Proyecto 865 armonización de las relaciones ecosistema - Cultura pero con la especificidad de reducir la vulnerabilidad frente al cambio climático. Recuperado de http://www.jbb.gov.co/jardin/institucional-inicio/plan-de-desarrollo-inicio

Leff, E. (2007). Aventuras de la epistemología ambiental: de la articulación de ciencias al diálogo de saberes. Mexico: Siglo XXI

Luhmann, N. (1996). Teoría de la Sociedad y Pedagogía. Barcelona: Paidós

MAVDT, \& MEN. (2002). Política Nacional de Educación Ambiental. Bogotá: MEN.

Mora, W. (2013). Educación en Ciencias y Educación Ambiental: Necesidad de una relación mutuamente beneficiosa. EDUCyT,134-148.

Morin, E. (1996). Introducción al pensamiento complejo. Barcelona: Gedisa.

Sauvé, L. (2010). Educación Científica y Educación Ambiental: Un cruce fecundo. Enseñanza de las Ciencias, 28(1), 5-18.

Sauvé, L. (Octubre. 2014). Educación científica y ecociudadanía. Retos contemporáneos. En VI Congreso internacional sobre formación de profesores de ciencias. Conferencia realizada el marco del Congreso. Bogotá.

SDA \& SDE. (2007). Política Pública Distrital de Educación Ambiental. Bogotá: SDA.

SDA. (2014). Proyecto 131 Participación ciudadana y educación ambiental como instrumentos de gestión para la apropiación social de los territorios ambientales del Distrito Capital. Recuperado de http://ambientebogota.gov.co/es/documentos-de-formulacion

Vasco Uribe, E.E., Martínez Boom, A. y Vasco Montoya, E. (2008) Educación, Pedagogía y Didáctica. Una perspectiva Epistemológica. En: G. Hoyos Vásquez (Ed), Filosofía de la Educación. Enciclopedia Iberoamericana de Filosofía (pp. 99-127) Madrid: Trotta.

Zambrano et.al. (2013). Las líneas de investigación en Educación en Ciencias en Colombia. EDUCyT, (7), 134-148. 